Horizons philosophiques

\title{
Le héros bergsonien et la manifestation du sens de l'être
}

\section{Henri Bah}

Volume 15, numéro 1, automne 2004

En quête du sujet

URI : https://id.erudit.org/iderudit/801276ar

DOI : https://doi.org/10.7202/801276ar

Aller au sommaire du numéro

\section{Éditeur(s)}

Collège Édouard-Montpetit

\section{ISSN}

1181-9227 (imprimé)

1920-2954 (numérique)

Découvrir la revue

\section{Citer cet article}

Bah, H. (2004). Le héros bergsonien et la manifestation du sens de l'être. Horizons philosophiques, 15(1), 53-65. https://doi.org/10.7202/801276ar d'utilisation que vous pouvez consulter en ligne.

https://apropos.erudit.org/fr/usagers/politique-dutilisation/ 


\section{Le héros bergsonien et la manifestation du sens de l'être}

Henri Bergson a proposé une métaphysique qui mérite d'être explorée et précisée si nous voulons asseoir une réflexion sur le sens à donner à notre être au monde. "La philosophie, écrit Bergson, est avant tout la science de la vie ${ }^{\prime}$. Penser intuitivement la vie, en dévoiler l'être et le sens, tel est l'objectif qu'il assigne à la philosophie. Or, l'intuition de la durée est à la fois le point de départ, le centre et le point d'arrivée de la doctrine bergsonienne. Cette intuition traverse et tisse la pensée bergsonienne. Sa philosophie de la durée est, avant tout, une philosophie de la vie. Lorsqu'il appréhende la vie par la méthode intuitive, cette vision directe de la vie par l'esprit lui permet d'approfondir la nature de la vie et d'en découvrir le sens. Elle révèle que la vie est durée ou que la durée est la vie. Cet "axiome» bergsonien permet d'éclairer la question du sens de la vie.

Et pourtant, dans la sphère de la philosophie, la question du sens de la vie a disparu au point que son simple souvenir semble désuet. En effet, dans notre univers sécularisé, nous assistons à l'oubli du sens. Car, si hier la question du sens de l'être était prise en charge par la foi et la philosophie, elle apparaît comme caduque voire ridicule aux yeux de l'homme contemporain. Or, durant des millénaires, cette question fut au cœur de la réflexion philosophique. Plus près de nous, Leibniz et, après lui, Heidegger, en faisaient la question fondamentale et première de la métaphysique. S'inspirant de Leibniz, Heidegger reprenait la question du sens en ces termes : «Pourquoi donc y a-t-il l'étant et non pas plutôt rien2?". Le constat est donc clair mais triste. C'est que la question du sens de notre vie s'avère aujourd'hui être un luxe intellectuel. Tout au plus, cette question n'effleure notre esprit que pendant des circonstances de notre déchéance comme la mort. Lorsque la mort nous frappe, nous ne pouvons retenir la question «Pourquoi»?

Or, l'atrocité des conflits de tout genre, qui déchirent notre univers actuel et qui menacent sérieusement l'avenir de l'humanité et l'humanité de l'avenir, exige que l'on se pose sérieusement la question du sens de notre vie. Mais dans quelle direction cette question peut-elle être orientée pour retrouver justement ce «sens du sens"? Mieux, comment retrouver le sens de notre vie dans une existence radicalement détachée de son origine? Si tant est que 
«Dieu est mort»; si la religion pourvoyeuse de sens ne peut plus nous guider; si la transcendance est bannie, comment donner du sens dans nos vies?

L'analyse qui va suivre se propose d'indiquer une piste expérimentale ou "expérimentable» pour chacun, afin de retrouver le sens de l'être de notre vie. À défaut d'une transcendance verticale, nous pensons qu'une transcendance horizontale dans la vie et en direction de ceux que Bergson nomme "les héros ${ }^{3}$ ", ceux-là même qui ont su retrouver l'impulsion originelle de l'élan de la durée, peut donner du sens à nos vies afin de comprendre nos malheurs et nos joies.

\section{La durée : l'être de la vie}

Bien que diversement abordée, la question du sens de l'être a été au centre de nombre de pensées philosophiques, depuis la période antique jusqu'au $20^{\circ}$ siècle, en passant par le $18^{\theta}$ siècle. Elle s'est estompée dans notre univers dominé par le progrès de la science. En effet, la science décrit ou explique partiellement le monde tel qu'il apparaît sous notre regard intéressé. La question radicale du "pourquoi»?, celle du sens, c'est-à-dire de ce que le monde est en réalité, n'est pas de son ressort. Seules la philosophie et, dans une certaine mesure, la religion cherchent à nous livrer le sens de la vie.

Mais si le religieux élève son regard vers Dieu, l'être absolu qui met fin à son angoisse, vers quel horizon sublime le philosophe peut-il orienter le sien, dans notre univers actuel, pour retrouver le sens de la vie?

La réponse est, à notre avis, contenue dans la question elle-même : la vie. La vie est justement là, elle-même pour nous livrer son sens, son être. Pourtant, cette réponse appelle, nous le savons, une objection : l'objection nietzschéenne que Luc Ferry explique largement :

Pour juger la vie, il faudrait pouvoir adopter sur elle une situation d'extériorité; pouvoir poser, hors d'elle, les termes de références auxquels s'adosser pour porter un jugement. II faudrait supposer une sphère transcendante idéale, un Audelà, une distance à partir de laquelle il y aurait quelque signification à émettre des évaluations. Or, c'est là l'illusion suprême, l'illusion par excellence de toute religion. L'homme 
est un vivant parmi d'autres, il appartient de plain-pied à la vie, il est immanent à elle et c'est pourquoi ses prétendus jugements ne sont que des symptômes des émanations inconscientes d'un certain type d'existence 4 .

L'objection est certes pertinente. Mais nous pensons qu'il faut ici faire la part des choses. Effectivement, l'intelligence, cette chose du monde la mieux partagée, a été surexploitée ou du moins surestimée par la philosophie. On a longtemps pensé qu'elle (l'intelligence) était le chemin d'accès à la vérité de toute réalité. Or, elle n'est qu'un chemin. Et comme telle, elle ne peut mener partout. De ce point de vue, Nietzsche et, avant lui, Kant ont raison de critiquer la vanité de l'intelligence et son usage utopique. Bergson reconnaîtra lui aussi que "notre pensée, sous sa forme purement logique est incapable de se représenter la vraie nature de la vie, la signification profonde du mouvement évolutif. (...) Déposée, en cours de route, par le mouvement évolutif, comment s'appliquerait-elle (l'intelligence) le long du mouvement évolutif lui-même ${ }^{5}$ "? Tous s'accordent donc à reconnaître que la vie déborde l'intelligence pour que celle-ci la pense. L'intelligence est, pour ainsi dire, dans une situation d'incompréhension naturelle de la vie.

Conscient des limites et de la nature même de l'intelligence, nous ne saurions donc l'utiliser pour interroger la vie afin qu'elle nous livre son être et son sens. Nous en appelons, par conséquent, à l'intuition au sens bergsonien du terme. II s'agit de cette faculté qui, différente de l'intelligence par nature, nous permet de comprendre la réalité tandis que l'intelligence éclaire notre action. L'intuition est contact immédiat avec la réalité qui dure. Par cette faculté, l'homme se place dans l'objet lui-même et en saisit le mouvement de vie. Avec Bergson, philosopher consiste donc en un effort intuitif pour se fondre dans le tout, couler avec lui, saisir le mouvement en lui, en soi. Ce tout n'est rien d'autre que la vie. Penser intuitivement, c'est donc penser la vie. "Nous appelons ici intuition la sympathie par laquelle on se transporte à l'intérieur d'un objet pour coïncider avec ce qu'il a d'unique et par conséquent d'inexprimable ${ }^{6}$ ».

Nous avons donc eu tort de vite condamner l'homme à l'incapacité naturelle d'appréhender la vie, comme si nous n'étions que pure intelligence, comme s'il n'y avait pas une nébulosité vague d'intuition autour de l'intelligence. Bergson pense donc que l'esprit humain est capable de saisir la vie parce qu'il y a en lui, autre chose 
que l'intelligence. Ainsi, nous amène-t-il, dans L'évolution créatrice, à interroger intuitivement la vie, c'est-à-dire à l'appréhender, à sympathiser et à coïncider avec elle pour en saisir l'être profond. La philosophie est, à ses yeux, la science de la vie capable de nous fournir une théorie de la vie qui se démarque de la théorie de la connaissance fournie par la science.

Comment l'intuition peut-elle appréhender la vie? «L'existence dont nous sommes le plus assurés et que nous connaissons le mieux est incontestablement la nôtre ${ }^{7}$ '. Je suis certain d'exister. Notre vie intérieure grâce à laquelle et avec laquelle nous affirmons une telle certitude est de nature constamment changeante. À y voir de près, «j'existe» est synonyme de «je change indéfiniment». En effet, je passe, sans rupture, mais continuellement d'un état à un autre. Des états de conscience défilent sans cesse qui colorent ma vie intérieure. Je pense à telle chose et à telle autre, j'ai chaud puis j'ai froid, je suis triste, gai, inquiet, je travaille, je ne fais rien, etc. Cette expérience, approfondie, nous amène à constater encore que l'état lui-même est déjà du changement. Les états de conscience "se continuent les uns les autres en un écoulement sans fin 8 ". C'est dire que ma personnalité, qui est faite de ces états, elle aussi change sans cesse. Qui plus est, ce changement est le déroulement ou le dévoilement imprévisible de nouveautés. Pris isolément (encore même si cela était possible), ces états de conscience sont de pures qualités et non des grandeurs quantitatives. Car on ne saurait les mesurer, les délimiter sans les dénaturer. Ils sont comme les notes de la mélodie qui sont de pures qualités se pénétrant les unes les autres et dont l'osmose crée la mélodie. "Les faits de conscience, même successifs, se pénètrent, et dans le plus simple d'entre eux peut se réfléchir l'âme entière ${ }^{9}$ \%.

II faut encore insister sur le fait que cette saisie intuitive n'est pas le fait de l'intelligence. Car là où l'intuition saisit du dedans, une succession sans distinction, une pénétration mutuelle, une solidarité dont chacun est représentatif du tout, l'intelligence n'y verra que des éléments à isoler, à opposer et à recomposer. Georges Canguilhem reconnaîtra bien lui aussi, après Bergson, cette attitude de l'intelligence : «Décomposer, réduire, expliquer, identifier, mesurer, mettre en équations, ce doit bien être un bénéfice du côté de l'intelligence puisque, manifestement, c'est une perte pour la jouissance ${ }^{10}$ ". Si notre vie intérieure est un pan de la vie en général, elle ne doit donc pas être interrogée dans les laboratoires de l'intelligence. 
Nous venons ainsi de vivre ou de revivre l'expérience de la vie intérieure. II faut en tirer les leçons. II ressort de cette expérience que si la durée est "une succession de changements qualitatifs qui se fondent, qui se pénètrent, sans contours précis, sans aucune tendance à s'extérioriser les uns par rapport aux autres 11 », elle constitue alors le mode d'existence de notre vie intérieure. La durée est l'être de la vie intérieure, c'est-à-dire sa substantialité, son essence, sa nature. Ce processus d'organisation qui caractérise notre vie intérieure est la durée. Mais ce n'est là qu'une conclusion partielle quant à l'objectif même de notre réflexion. Interrogeons encore le monde extérieur afin qu'il nous livre son mode d'être et, alors seulement, nous pourrons conclure de l'essence de la vie en général.

Plus manifeste que dans la vie intérieure, est la succession dans le monde extérieur. Prenons l'exemple de l'être vivant. La caractéristique première de cet être, c'est qu'il est vivant. II est donc traversé par la vie. Mais quelles sont les modalités de la vie en lui? Le corps vivant n'est pas un corps comme les autres. II se compose de parties hétérogènes qui se complètent les unes les autres. Le corps vivant accomplit ainsi des fonctions qui s'impliquent mutuellement. Par exemple, la fonction de reproduction est liée à celle de nutrition, à celle de la circulation du sang (pour les animaux et les hommes), à celle de la respiration, et vice versa. L'être vivant est toujours en voie de réalisation, en perpétuel devenir. C'est ce qui nous fait parler de l'évolution, du vieillissement d'un homme ou de tout autre vivant. Dans le cas de l'homme en l'occurrence, nous procédons, avec l'intelligence, à des découpages dans ce flux de son progrès et nous distinguons des phases comme : l'œuf, l'embryon, l'enfant, l'adolescent, l'adulte et le vieillard. En réalité, ce ne sont là que des hétérogénéités du même flux de son changement sans cesse continu. Ces hétérogénéités sont continues les unes dans les autres. Où est en fait le point qui sépare radicalement, du moins biologiquement, ces phases les unes des autres?

Même les corps inorganisés, qui ne semblent pas "vivre», connaissent, en réalité, la traversée de la vie. Mon morceau de sucre fond selon une durée qui est la sienne et non selon mon empressement. Naissance, croissance, vieillissement, mort, putréfaction, constituent le mode d'existence de tout ce qui est. Le cas est donc que le monde extérieur, tout comme la vie intérieure, déroulent une histoire, c'est-à-dire une succession où il n'y a pas de 
répétition, où tout est unique et porte en lui la représentation de tout le passé.

On pourrait dès lors conclure que «la durée est immanente au tout de l'univers. L'univers durexii" . Chaque moment de notre vie apparaît comme une création continue qui prolonge l'acte originel. Pour l'être conscient, exister consistera alors à changer, à mûrir, à se créer et à créer indéfiniment. La durée est l'étoffe dont est faite notre vie. Commentant le propos bergsonien, Madaule écrit avec justesse : "La vie est durée. La conscience, en effet, qui dure, est coexistence avec la vie ${ }^{x_{i i i}}{ }_{\text {}}$. La sagesse devrait alors consister à savoir que rien dans ce monde n'est permanent. Toute chose est périssable. La seule chose qui est éternelle, c'est "l'impermanence».

Si telle est l'essence ou l'être de la vie, comment l'homme peut-il donner du sens à sa vie qui n'est qu'une dimension de la vie en général? Quel sens donner à notre être au monde pour justement participer à ce mouvement créateur et continu de la durée?

Dans un monde sécularisé, où la raison, après ses promesses de bonheur, nous a trahis, nous pensons qu'à défaut d'une transcendance horizontale, une transcendance verticale en direction de ces hommes exceptionnels qui nous fascinent, défient la raison et l'inquiètent, nous permettrait de faire l'expérience sensible du sens de l'existence.

\section{L'expérience du héros comme sens de l'être}

Avant de dégager le sens de la vie, il nous fallait en dévoiler l'Être. Nous venons de montrer que la durée, création continue d'hétérogénéités solidaires les unes aux autres, est l'être de la vie. C'était donc là un pas considérable dont il nous faut à présent tirer les leçons et les conséquences afin de répondre à notre préoccupation principale.

Quel sens donner à cette vie qui est durée? Analysant la «structure personnelle» du mot "sens", Luc Ferry arrive à la conclusion suivante : ce mot implique en lui-même l'idée d'une volonté, d'une intention. C'est pourquoi, nous disons le plus souvent, pour désigner le sens, "qu'est-ce que cela veut dire?». Dans le sens, il y a donc l'idée d'une intention qu'un sujet veut communiquer aux autres. II ne nous viendrait nullement à l'esprit (à moins d'être superstitieux), de demander "le sens" d'une chaise, d'un arbre ou d'un mur. Par contre, nous pouvons le faire pour l'expression d'un visage, pour un panneau routier. Ferry en tire alors l'axiome suivant : «N'a pas ou ne fait pas 
sens tout ce qui n'est pas l'effet d'une volonté, tout ce qui n'est pas en quelque façon manifestation d'une subjectivité ${ }^{14}$ ».

S'interroger sur le sens de l'être de la vie, reviendrait alors à chercher à savoir l'intention, la volonté qui se dégage de cet être de la vie. Mais si pour saisir l'être de la vie, il nous a fallu interroger les pôles de la vie que sont notre intériorité et le monde extérieur, comment saisir alors la "volonté» ou l'intention que cet être nous communique?

Dans L'évolution créatrice, Bergson nous disait d'abord que la vie entière émane d'un élan originel, d'un élan vital qui, dans une impulsion initiale, s'est propagé dans des directions divergentes. II précise que cet élan part lui-même d'un centre. "Je parle d'un centre d'où les mondes jailliraient comme les fusées d'un immense bouquet, pourvu toutefois que je ne donne pas ce centre pour une chose, mais pour une continuité de jaillissement. Dieu, ainsi défini, n'a rien de tout fait; il est vie incessante, action, liberté ${ }^{15}$ ". Dieu serait donc l'auteur de ce mouvement. Mais Bergson ajoute bien que, le Dieu dont il est question n'est pas du tout fait, l'être stable achevé qui regarderait ce mouvement se poursuivre. Ce n'est pas le Dieu de nos religions. II est lui-même vie incessante. Nous dirions, selon l'expression spinoziste, qu'il est nature naturante, nature naturée. Quelle que soit notre manière de l'envisager, toute réflexion sur la vie débouche sur ce mystère qu'à l'origine de la vie, il y a un acte créateur du créateur. La transcendance est immanente à l'esprit humain. Comme le dit Ferry, «lorsque j'ouvre les yeux sur le monde, il m'apparaît de manière indiscutable comme non créé par ma propre conscience. J'ai donc, en moi (immanence), le sentiment contraignant du "hors de moi» (transcendance) ${ }^{16}$,

Ensuite, dans Les deux sources, Bergson complète sa «découverte". L'élan vital qui frise dans la matière est parvenu à l'homme qui brise la chaîne. Avec l'homme, le sens de l'évolution apparaît. En effet, des âmes exceptionnelles ont existé qui sont des ruisselets entre lesquels se partage le grand fleuve de la vie. Ces hommes, qui ont mené notre vie, notre expérience quotidienne, se distinguent par leur expérience. Ayant été en contact avec la source originelle d'où jaillit la durée, ils veulent communiquer le message de vie à l'humanité.

Nous en arrivons ainsi au cœur même de notre réflexion. Nous pensons que comprendre le sens de la vie ou donner du sens à nos vies reviendrait à capter le message de ces personnes 
exceptionnelles que Bergson nomme les héros. En dépit de leur diversité et du caractère individuel de leurs expériences respectives, les héros arrivent à une conclusion unique : l'amour est ce sentiment qui donne un souffle au mouvement de vie. L'intention de la vie (son sens), c'est l'amour de l'homme pour l'homme. Il faut être assez sourd ou être sous le poids de la matière pour ne pas entendre ce message de l'intuition créatrice que véhicule le héros.

Déjà dans le Cratyle, Socrate répondant à la question de Hermogène, relative au "sens" du mot héros, disait ceci : "Ce nom dont la forme a été légèrement détournée, indique la naissance due à l'amour. ( ...) Ne sais-tu pas que les héros sont des demi-dieux? Considère encore ce nom à la lumière de l'ancienne langue attique, et tu t'en rendras mieux compte : tu verras qu'il a été dérivé du nom de l'amour (érôs) auquel les héros ont dû la naissance ${ }^{16 " . ~ M a i s ~ l ' a m o u r ~}$ présente tant de visages qu'il est nécessaire de faire ici la part des choses, lorsque nous parlons de l'amour comme sens de la vie qui se réalise dans l'expérience du héros.

Éros, dont parle assez largement Platon, désigne le désir sexuel. Exprimé dans la passion amoureuse, il traduit l'idée d'un manque. On aime l'autre en vue de le "dévorer". Une fois ce désir assouvi, il s'éteint jusqu'à ce qu'il se rallume. II y a donc dans cet amour, à la fois l'idée de manque et de satisfaction. Freud en dévoilera d'ailleurs l'instinct d'agressivité voilé. Eros s'abîme ou se transforme le plus souvent en thanatos. Cet amour ne saurait être, pour des raisons évidentes, le sens de la vie. Qui ne voit pas que ce sentiment seul unit les êtres, mais qu'il peut à lui seul aussi les désunir?

Philia, dont Aristote se fait le porte-parole, renvoie à l'amitié. II s'exprime non pas dans le manque et la consommation, mais dans la joie qui naît de la simple présence, de la seule existence de l'être aimé. C'est l'amour-joie, la joie d'aimer et d'être aimé. II ne se réduit donc pas à la simple passion. Cet amour, bien que supérieur à Éros, est cependant limité. Nous ne pouvons l'éprouver pour plus de cinq personnes. Nous n'avons généralement qu'un seul ou au plus deux amis.

L'amour Agapè, qui trouve ses lettres de noblesse dans les Évangiles, désigne un amour désintéressé, gratuit. C'est à cet amour que le Christ nous convie. II nous demande de l'étendre même à ceux qui nous sont indifférents voire à nos ennemis. "Aimez vos ennemis, faites du bien à ceux qui vous haïssent, bénissez ceux qui vous maudissent ${ }^{18}$ ». 
Débarrassé de ses dogmes religieux, de ses contraintes théologico-éthiques, cet amour est, pour nous, le sens de la vie. Nous ne sommes de ce fait pas d'avis avec Luc Ferry lorsqu'il affirme que : «Agapè rendrait la morale superflue, cet amour est si gratuit, si désintéressé qu'il semble inaccessible à l'humain ${ }^{19}$ ". Un tel jugement est la conséquence de ce que nous voulons toujours appréhender la vie dans les filets de l'intelligence. II résulte de l'incapacité naturelle de l'intelligence à saisir l'élan de vie qui est aussi l'élan d'amour.

Mais, répétons-le, avec l'intuition, avec l'âme mystique, nous pouvons reprendre l'expérience des héros, qui est une expérience de l'amour-créateur. Même si le vrai mysticisme est rare et se veut une expérience difficile, la philosophie n'a pas une autre méthode, en dehors de l'intuition mystique, pour aborder le problème de l'existence. Seul le mysticisme nous fournit, expérimentalement, le moyen d'aborder le problème du sens de l'existence. D'ailleurs ce qui est rare et exceptionnel n'est pas pour autant impossible. II faut simplement avoir l'audace et l'énergie de reprendre leur expérience comme Stanley en a eu pour aller retrouver Livingstone. Leur expérience révèle qu'un élan d'amour les portait à élever l'humanité jusqu'à Dieu, de sorte à parfaire la création. Bergson, traduisant cette expérience du héros, écrit ceci : "Car l'amour qui le consume n'est plus simplement l'amour d'un homme pour Dieu, c'est l'amour de Dieu pour tous les hommes. À travers Dieu, par Dieu, il aime toute l'humanité d'un divin amour20". II s'agit, chez Bergson, si peu du Dieu auquel pensent la plupart des hommes. D'ailleurs, ce qui importe ici, c'est moins la nature de ce Dieu que le résultat de l'expérience. Car une âme capable de cette expérience ne se demande même pas si le principe avec lequel elle entre en communion intime est la cause transcendante de toutes choses ou si ce n'en est que la délégation terrestre. À strictement parler, le mot religion n'a plus sa place ici. II n'y a qu'amour et joie.

Pourquoi pensons-nous que cet amour est le sens de la vie? Le détour par la durée nous a laissés découvrir qu'elle est flux continu, création et solidarité d'hétérogénéités. Or, l'amour, tel qu'exprimé par les mystiques comme le Christ, Socrate et autres Arahants du bouddhisme, traduit toutes ces qualités de la durée. Cet amour est créateur parce qu'il amène les héros eux-mêmes et ceux qui les ont rencontrés à se recréer. lls ne demandent rien, et pourtant ils obtiennent. Leur seule présence suffit pour nous recréer. Le croyant qui a connu le Christ dit, avec beaucoup de sens : «je suis né 
de nouveau", comme si le Christ venait de l'amener à l'existence. L'expérience du mystique continue l'acte créateur de la durée. Par son amour, le héros se fait co-créateur de l'acte créateur.

Le caractère de flux continu de la durée se retrouve dans cet amour que le mystique propage, sans fin et sans discrimination, sur tout ce qui existe. Comme une fumée qui prend sa source dans un foyer, se propage dans l'atmosphère et contamine toute la nature, cet amour est sans borne. L'amour du mystique embrasse l'humanité entière, s'étend aux animaux, aux plantes et à toute la nature. Le Christ n'avait pas de frère particulier. L'homme était son frère. Le christianisme, qui a inventé l'expression assez suspecte de ufrère en Christ», dénature cet amour du Christ pour tout homme. Car dans cette expression, ce n'est plus l'autre en tant que tel qui est objet d'amour mais le chrétien potentiel.

La solidarité des hétérogénéités se voit à travers l'union, la solidarité ou la fraternité que le mystique éprouve et qu'il manifeste pour tous les hommes, en dépit de sa différence raciale, culturelle, religieuse, et politique avec ces hommes. Cette solidarité s'explique par le fait que le mystique a compris l'unité de la source au-delà de la multiplicité des termes. II y a certes hétérogénéités, mais aussi et surtout identité de source. On ne s'étonnera donc pas de constater assez souvent des traits de ressemblance entre la plante, l'animal et même l'homme. II se trouve que torpeur, instinct et intelligence sont des directions divergentes de l'évolution de la vie.21 Pourquoi donc le mystique n'aimerait-il pas ces «enfants" du même élan? II n'y a que l'intelligence qui, n'ayant pas saisi cette unité de source, puisse céder à la séparation, à la discrimination, au racisme et à la xénophobie. Certaines critiques de la frénésie technologique nous démontrent bien le danger qui menace les hommes dans leur destruction de la nature. On n'a pas hésité à parler de la communauté de destin de l'homme avec la nature. C'est que ces manifestations éparpillées de la vie doivent, à la communauté de leur origine, d'être complémentaires les unes aux autres.

II ressort donc de cette analyse qu'il y a une différence de nature entre l'amour du héros pour l'humanité et les autres formes d'amour. En outre, cet amour renferme toutes les qualités de l'être même de la vie. C'est pourquoi nous n'hésitons pas à faire de cet amour le sens ultime de la vie. En clair, la question du sens de la vie débouche sur celle de l'éthique. Elle pourrait alors être comprise plus simplement ainsi : comment agir pour réaliser ou pour donner du sens à nos vies? 
Dans la mesure où une morale ne peut être supérieure à la vie, notre agir ne peut retrouver le sens de la vie qu'en reprenant l'expérience des héros qui en expriment le mieux "l'intention». L'expérience du héros est la manifestation sensible du sens de la vie. Même Zarathoustra de Nietzsche, recommandait à ses disciples : "comme moi ramenez sur terre la vertu égarée dans son vol (...) afin qu'elle donne à la terre son sens, un sens humain ${ }^{22}$ ». C'est dire que seule la morale, avec en son centre la vertu, est le sens de la vie.

Mais face à ce sens assez sublime, la conscience est toujours là qui nous souffle à l'oreille, qu'une telle morale n'est pas faite pour l'homme ordinaire. II n'y a eu qu'un seul Jésus-christ, un seul Socrate ou plus près de nous, une seule Mère Térésa.

Nous ne croyons pas en un tel pessimisme, en une telle froideur de la conscience. L'homme est capable d'une âme close, d'une âme qui s'ouvre et d'une âme ouverte. Autrement dit, être tout à fait homme, c'est être un peu moins qu'homme et un peu plus qu'homme. La montée de la barbarie humaine, au Liberia, en Ex-Yougoslavie, au Rwanda, en Irak, a largement démontré que l'homme est vraiment capable d'être moins qu'homme. Heureusement, face à cette frénésie, une autre, de force antagoniste, monte à travers le monde qui nous dit que l'homme est aussi plus qu'homme. En effet, «toute action prolongée dans un sens amène une réaction en sens contraire ${ }^{23}$ ". C'est là une loi, de l'évolution de la vie, à laquelle l'évolution psychologique et sociale de l'homme ne saurait échapper.

Les actions humanitaires, même si elles ne sont pas des actions d'humanité, comme celles des héros, élèvent l'univers vers l'humanité. Cet élan d'actions caritatives, que nous voyons se développer à travers la sphère politique et celle des ONG, est dans une certaine mesure porteur de sens. II exprime l'amour de l'homme pour l'homme, du moi pour autrui. Écrivant à ses parents depuis la Somalie en mars 1982, un membre d'une ONG caritative disait : "Le travail est extrêmement exténuant mais je deviens follement heureux d'être médecin et je réalise enfin que tout ce qui m'a fait râler pendant sept ans sert à quelque chose. L'expérience que je vis ici est fantastique. Je réapprends à vivre...24». Ces personnes ordinaires, qui éprouvent du bonheur à sauver des vies au prix de leur vie, pensent avoir ajouté un peu de sens à leur vie. En effet, c'est reconnaître un sens supérieur à sa propre existence que de consentir volontairement de tels sacrifices. Ainsi, à défaut de faire 
ce qu'ont fait les grands mystiques, font-ils comme ils ont fait. Si tant est que entre l'âme close et l'âme ouverte il y a l'âme qui s'ouvre, ces actions humanitaires sont déjà celles d'une âme qui s'ouvre. Comprendre qu'on ne peut pas aimer ses enfants et rester insensible aux malheurs qui frappent d'autres enfants, fût-ce à l'autre bout du monde, c'est comprendre le sens de la vie, et donner sens à sa vie. C'est face à la mort et au mal sous toutes ses formes qu'apparaît le sens de la vie. Nous pensons par ailleurs que, nos défis de paix dans le monde, de mondialisation (des cultures et des économies) ne sont que l'expression de cet amour auquel les mystiques et les héros nous convient. Ces nobles ambitions ne sauraient de ce fait voir le jour si nous ne répondons pas, du fond du cœur, à leur appel.

L'humanisme bergsonien, dans lequel nous voyons le sens de la vie, est loin d'une transcendance verticale. II ne s'agit pas de s'élever, mais de rester dans la vie elle-même, de s'en laisser imprégner profondément. Si transcendance il y a, elle relie les êtres humains ou vivants entre eux. Cet humanisme s'illustre à juste titre par l'expérience vivante des mystiques. La vie du Christ et des autres mystiques doit être perçue comme un message porteur d'un sens symbolique : la vie est amour. L'amour maternel si frappant chez les hommes, si touchant chez certains animaux, observable chez la plante pour sa graine, n'est pas un mystère. C'est là que gît la réalité du sens de la vie. II est amour. Cet amour est lui-même création continue.

\section{Dr Henri Bah \\ Philosophie, université de Bouaké, Côte d'Ivoire}

1. Henri Bergson, Mélanges, Paris, PUF, 1972, p. 1090.

2. Heidegger, Introduction à la métaphysique, Paris, Gallimard, 1967, Chapitre I, p.13. Cette question heideggerienne s'inspire de celle de Leibniz. Seulement, là où Leibniz employait l'expression "quelque chose», Heidegger, fidèle à sa distinction de l'étant et de l'être, écrit “l'étant". Quelle que soit sa forme, cette question radicale interroge, au fond, en direction du sens de la vie.

3. Henri Bergson, Les deux sources de la morale et de la religion, Paris, PUF, 1995, p. 29

4. Luc Ferry, L'homme-Dieu ou le sens de la vie, Paris, éd. Grasset, 1996, p. 230.

5. Henri Bergson, L'évolution créatrice, Paris, PUF, 1986, p. VI.

6. Henri Bergson, La pensée et le mouvant, Paris, PUF, 1993, p. 181.

7. Henri Bergson, L'évolution créatrice, p. 1. 
8. Ibid., p. 3.

9. Henri Bergson, Essai sur les données immédiates de la conscience, Paris, PUF, 1970, p. 73.

10. Georges Canguilhem, La connaissance de la vie, Paris, Vrin, 1998, p. 9.

11. Henri Bergson, Essai sur les données immédiates de la conscience, p. 77.

12. Henri Bergson, L'évolution créatrice, p.11.

13. Madeleine Madaule, Bergson, Paris, PUF, 1967, p. 38.

14. Luc Ferry, op. cit., p. 35.

15. Henri Bergson, L'évolution créatrice, p. 249.

16. Luc Ferry, op. cit., p. 50

17. Platon, Cratyle, Paris, Flammarion, 1980, 398b

18. Bible, Nouveau Testament, Jean $6: 27-28$

19. Luc Ferry, op. cit., p. 162.

20. Henri Bergson, Les deux sources de la morale et de la religion, p. 247.

21. Henri Bergson, L'évolution créatrice, p. 99.

22. F. Nietzsche, Ainsi parlait Zarathoustra, Paris, éd. 10/18, 1958, p. 71\&72.

23. Henri Bergson, Les deux sources de la morale et de la religion, p. 311.

24. Extrait de "Lettres sans frontière" Luc Ferry, L'homme-Dieu ou le sens de la vie, $p$. 204. 